\section{Carbon dioxide enterography: a useful method for double-balloon enteroscopy-assisted ERCP}

Development of double-balloon enteroscopy-assisted endoscopic retrograde cholangiopancreatography (DB-ERCP) has enabled endoscopic treatment of pancreatobiliary disease in patients with a surgically altered gastrointestinal anatomy [1].
However, scope insertion requires experience because of the maze-like gastrointestinal tract [2]. Intraluminal injection of indigo carmine to identify the afferent loop of Roux-en-Y anastomosis [3] may cause susceptibility to peristalsis and is unsuitable for complex reconstruction. To develop a smooth insertion method, we used a negative contrast technique with carbon dioxide to confirm the correct tract, termed $\mathrm{CO}_{2}$ enterography, and we present case results here.

An 86-year-old man had previously undergone pancreatoduodenectomy had a suspected anastomotic stricture of the choledochojejunostomy. Fig. 1 a shows the double-balloon enteroscope at the jejunojejunostomy, after which the operator inserted the tip of the scope into one of
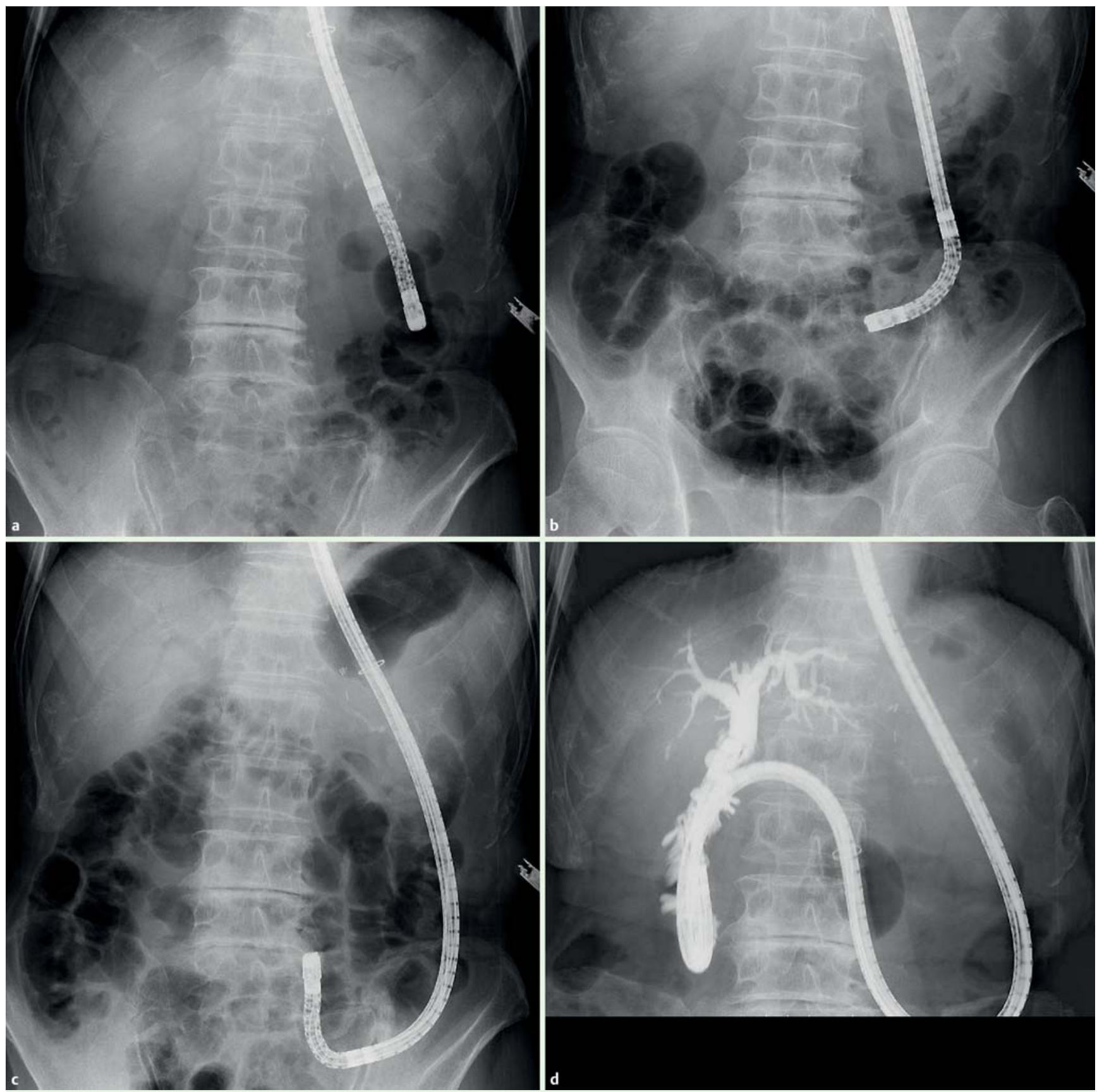

Fig. 1 a Double-balloon enteroscope at the jejunojejunostomy in an 86-year-old man who had previously undergone pancreatoduodenectomy and had a suspected anastomotic stricture of the choledochojejunostomy. b Fluoroscopy revealed $\mathrm{CO}_{2}$ directed to the anal side, indicating the incorrect tract. $\mathbf{c}$ Insertion into another tract. $\mathrm{CO}_{2}$ enterography revealed the correct tract for the choledochojejunostomy. $\mathbf{d}$ After reaching the target site, cholangiography showed no strictures. 

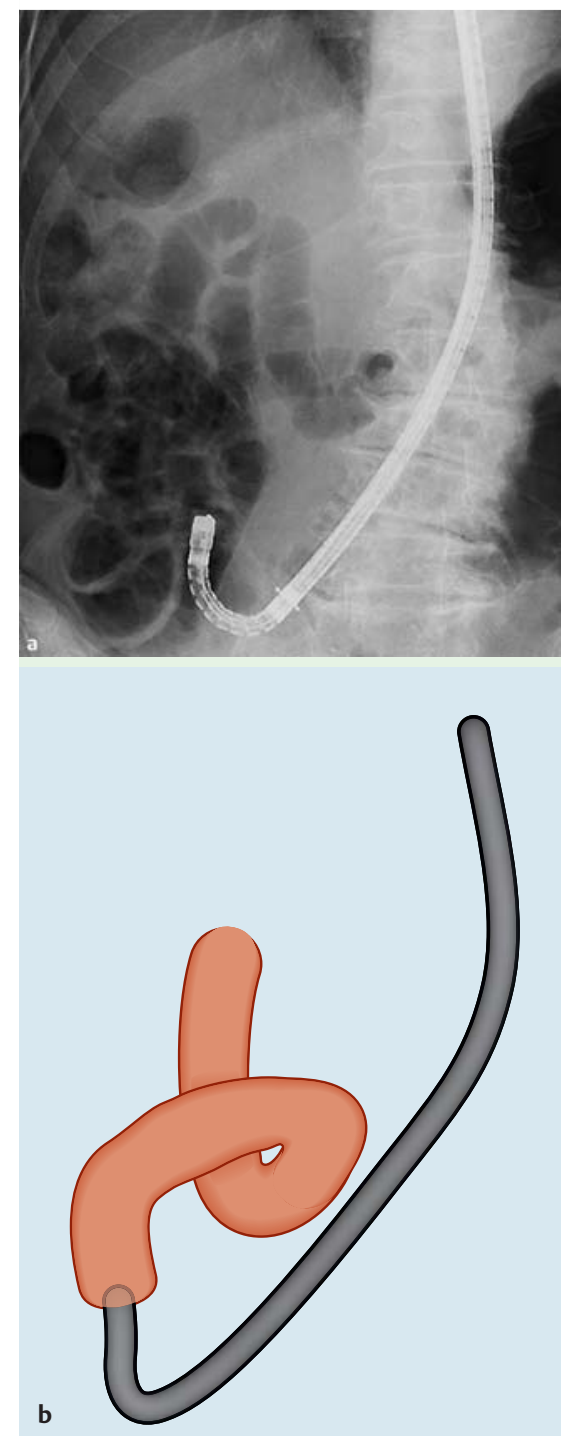

Fig. 2 An 84-year-old man underwent a distalgastrectomy with Billroth II reconstruction. a $\mathrm{CO}_{2}$ enterography confirmed the correct tract with Billroth II reconstruction. b Schema. Orange area corresponds to the afferent loop. the two tracts and injected $\mathrm{CO}_{2}$ under the obstruction caused by scope balloon inflation. Fluoroscopy revealed $\mathrm{CO}_{2}$ directed to

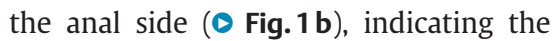
incorrect tract. $\triangle$ Fig. $1 \mathrm{c}$ shows insertion into another tract, after which $\mathrm{CO}_{2}$ enterography revealed the correct tract for the choledochojejunostomy. After reaching the target site, cholangiography showed no strictures ( $\bullet$ Fig. 1 d). In an 84-yearold man who underwent a distal gastrectomy with Billroth II reconstruction, $\mathrm{CO}_{2}$ enterography confirmed the correct tract ( $\mathbf{F i g} .2$ ). $\mathrm{CO}_{2}$ enterography was suitable for various surgically altered gastrointestinal tract cases.

We retrospectively investigated target site arrival times with $(n=39)$ and without $(\mathrm{n}=16) \mathrm{CO}_{2}$ enterography in post-surgical patients, excluding those with Billroth I reconstruction. The average time was significantly shorter in the $\mathrm{CO}_{2}$ enterography group (26 vs. 38 minutes, $P=0.026$ ). No adverse events related to $\mathrm{CO}_{2}$ enterography were observed. Using $\mathrm{CO}_{2}$ enterography, the correct tract was easily identified without wasted effort from insertion into the incorrect tract. Thus we consider it useful for insertion in DB-ERCP cases.

\section{Endoscopy_UCTN_Code_TTT_1AR_2AK}

Competing interests: None
Nobuhiko Fukuba', Ichiro Moriyama², Shunji Ishihara', Takafumi Yuki ${ }^{3}$, Kousaku Kawashima ${ }^{1}$, Norihisa Ishimura ${ }^{1}$, Yoshikazu Kinoshita ${ }^{1}$

${ }^{1}$ Department of Internal Medicine II, Shimane University School of Medicine, Izumo, Japan

${ }^{2}$ Division of Cancer Center, Shimane University Hospital, Izumo, Japan

${ }^{3}$ Division of Gastrointestinal Endoscopy, Shimane University Hospital, Izumo, Japan

\section{References}

1 Shimatani M, Matsushita M, Takaoka M et al. Effective 'short' double-balloon enteroscope for diagnostic and therapeutic ERCP in patients with altered gastrointestinal anatomy: A large series. Endoscopy 2009; 41: 849-854

2 Shimatani M, Takaoka M, Matsushita M et al. Endoscopic approaches for pancreatobiliary diseases in patients with altered gastrointestinal anatomy. Dig Endosc 2014; 26 (Suppl. 01): 70-78

3 Yano T, Hatanaka H, Yamamoto $H$ et al. Intraluminal injection of indigo carmine facilitates identification of the afferent limb during double balloon ERCP. Endoscopy 2012; 44: E340-E341

\section{Bibliography}

DOI http://dx.doi.org/

10.1055/s-0034-1377943

Endoscopy 2014; 46: E587-E588

(c) Georg Thieme Verlag KG

Stuttgart · New York

ISSN 0013-726X

\section{Corresponding author}

\section{Nobuhiko Fukuba, MD}

Department of Internal Medicine II

Shimane University Faculty of Medicine

89-1 Enya-cho

Izumo

Shimane

Japan

Fax: +81-853-202187

fnat98@yahoo.co.jp 